\title{
Factorization breaking in diffractive photoproduction of dijets-a review
}

\author{
Gustav Kramer a \\ aII. Institut für Theoretische Physik, Universität Hamburg, \\ Luruper Chaussee 149, D-22761 Hamburg, Germany
}

I compare the latest $\mathrm{H} 1$ and ZEUS data on diffractive dijet photoproduction with next-to-leading order QCD predictions in order to see whether a rapidity gap survival probability of less than one is supported by the data. I find evidence for this hypothesis when assuming global factorization breaking for both the direct and resolved photon contributions, in which case the suppression factor would have to be strongly $E_{T}^{j e t}$-dependent, and when assuming factorization breaking for the resolved or in addition the related direct initial-state singular contribution only, where it would be independent of $E_{T}^{\text {jet }}$.

\section{Introduction}

Factorization breaking in diffractive dijet photoproduction in connection with next-to-leading order (NLO) QCD predictions has been investigated the first time in 2004 [1,2] by comparing to preliminary H1 data [3]. Since then numerious further preliminary studies have been presented by the $\mathrm{H} 1$ and ZEUS collaborations at various conferences and workshops. In the meantime, the final diffractive parton distribution functions (DPDFs), which have been determined by the H1 collaboration from the inclusive measurements of the diffractive structure function $F_{2}^{D}$, have been published [4]. These DPDFs constitute a necessary input for the NLO prediction of the dijet production cross sections. Also, both collaborations, H1 and ZEUS, have now published their final experimental data of the cross sections for diffractive dijet photoproduction $[5,6]$. Whereas $\mathrm{H} 1$ confirms in [5] their earlier findings, based on the analysis of their preliminary data and preliminary DPDFs, the ZEUS collaboration [6] reached somewhat different conclusions from their analysis. In particular, the H1 collaboration [5] obtained a global suppression of their measured cross sections as compared to the NLO calculation of approximately $R=0.5$. In addition they concluded that also the direct cross section together with the resolved one does not obey factorization. The ZEUS collaboration, on the other hand, concluded from their analysis $[6]$ that, within the large uncertainties of the NLO calculation and the differences in the DPDF input, their data are compatible with the QCD calculation, i.e. suppression could not be deduced from their data.

From the viewpoint of pertubative QCD (pQCD) the central problem for hard diffractive scattering processes, characterized by a large rapidity gap in high-energy collisions, is whether they can be factorized into non-perturbative PDFs of a colorless object (e.g. a pomeron) and perturbatively calculable partonic cross sections. This concept is believed to be valid for the scattering of point-like electromagnetic probes off a hadronic target, such as deep-inelastic scattering (DIS) or direct photoproduction [7], but has been shown to fail for purely hadronic collisions [7] in agreement with measurements of the CDF collaboration at the Tevatron [8]. Factorization is thus expected to fail also in resolved photoproduction, where the photon first dissolves into partonic constituents, before these scatter off the hadronic target. The separation of the cross section into these two types of photoproduction processes is, however, a leading order (LO) concept. At NLO of pQCD, they are closely connected by an initialstate (IS) singularity originating from the splitting $\gamma \rightarrow q \bar{q}$ (for a review see [9]), which may play a role in the way factorization breaks down in diffractive photoproduction [10]. The breaking 
of the resolved photoproduction component alone leads to a dependence of the predicted cross section on the factorization scale $M_{\gamma}[1,2,10]$. Since such a $M_{\gamma}$-dependence is unphysical, it must be restored also for the factorization breaking of the resolved part of the cross section, e.g. by modifying the IS singular direct part. A proposal how to achieve this has been worked out in [10] and has been reviewed already by $M$. Klasen at the Ringberg workshop in 2005 [11] and in the proceedings of the workshop on HERA and the $L H C$ of 2004-2005 [12] (see also [13]). Since from a theoretical point of view only the suppression of the resolved or in addition the IS singular direct component [10] can be justified, it is an interesting question whether the diffractive dijet photoproduction data actually show breaking of factorization, how large the suppression in comparison to no breaking will be, and whether the breaking occurs in all components or just in the resolved plus direct IS component. The value of the suppression factor or survival probability can then be compared to theoretical predictions [14] and to the survival probability observed in jet production in $p \bar{p}$ collisions at the Tevatron [8] and will be of interest for similar diffractive processes at the LHC.

With regard to the somewhat inconsistent results published by the $\mathrm{H} 1$ and ZEUS collaborations we made a new effort [15] to analyze the H1 [5] and the ZEUS [6] data, following more or less the same strategy as in our earlier work $[1,2]$ on the basis of the NLO program of $[1,2]$ and the new DPDF sets of Ref. [4] of the H1 collaboration. The $\mathrm{H} 1$ and the ZEUS dijet data cannot be compared directly, since they have different kinematic cuts. Specifically, in the H1 measurements [5] $E_{T}^{\text {jet1(2) }}>5$ (4) $\mathrm{GeV}$ and $x_{\mathbb{P}}<0.03$, and in the ZEUS measurements [6] $E_{T}^{\text {jet1(2) }}>7.5(6.5)$ $\mathrm{GeV}$ and $x_{\mathbb{P}}<0.025$ (these and all other variables used in this review are defined in $[1,2,15]$ and in the corresponding experimental contribution in these proceedings [16]. In order to establish a global suppression, i.e. an equal suppression of the direct and the resolved cross section, the absolute normalization and not so much the shape of the measured cross section is essential. This normalization depends on the applied kinematic cuts. Of course, the same cuts must be applied to the NLO cross section calculation. In case of a resolved suppression only, the suppression depends on the normalization of the cross sections, but also on the shape of some (in particular the $x_{\gamma}^{o b s}$, $E_{T}^{j e t 1}, M_{12}$, and $\bar{\eta}^{\text {jets }}$ ) distributions, and will automatically be smaller at large $E_{T}^{j e t 1}[1,2,9]$, since the resolved cross section decreases more strongly with increasing $E_{T}^{j e t 1}$ than the direct one. Distributions in $x_{\mathbb{P}}$ and $y$ (or $W$ ) are not sensitive to the suppression mechanism. The distribution in $z_{\mathbb{P}}$ is sensitive to the functional behavior of the DPDFs, in particular of the gluon at large fractional momenta and small scales.

Just recently, the $\mathrm{H} 1$ collaboration made an effort to put more light into the somewhat differing conclusions of the $\mathrm{H} 1$ [5] and ZEUS [6] collaborations by performing a new analysis of their data, now with increased luminosity, with the same kinematic cuts as in [5], i.e. the low$E_{T}^{j e t 1}$ cut, and the high- $E_{T}^{j e t 1}$ cut as in the ZEUS analysis [6]. The results have been presented at DIS 2008 [17]. We have performed a new comparative study of these H1 [17] and ZEUS data [6] to show more clearly the differences between the three data sets [18]. In this review I shall show a selection of these comparisons. The emphasis will be on how large the survival probability of the diffractive dijet cross section globally is and whether the model with resolved suppression only will also describe the data in a satisfactory way. In section 2 we show the comparison with the H1 data [17] and in section 3 with the ZEUS data [6]. In section 4 I present my conclusions.

\section{Comparison with recent $\mathrm{H} 1$ data}

The recent $\mathrm{H} 1$ data on diffractive photoproduction of dijets [17] have several advantages as compared to the earlier H1 [5] and ZEUS [6] analyses. First, the integrated luminosity is three times higher than in the previous H1 analysis [5] comparable to the luminosity in the ZEUS analysis [6]. Second, H1 took data with low- $E_{T}^{\text {jet }}$ and high$E_{T}^{j e t}$ cuts, which allows for a comparison with [5] and [6]. The exact two kinematic ranges are given in $[17]$. 

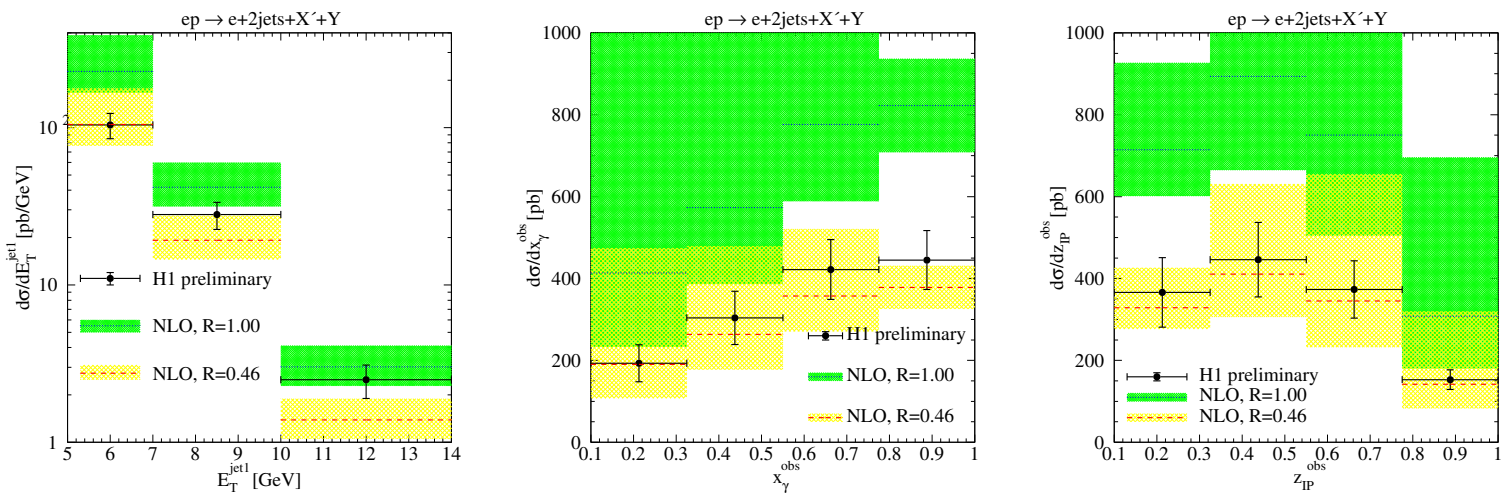

Figure 1. Differential cross sections for diffractive dijet photoproduction as measured by H1 with low- $E_{T}^{\text {jet }}$ cuts and compared to NLO QCD without $(R=1)$ and with $(R=0.46)$ global suppression as a function of $E_{T}^{j e t 1}(\mathrm{a}), x_{\gamma}^{o b s}(\mathrm{~b})$ and $z_{\mathbb{P}}^{o b s}(\mathrm{c})$ (color online).

The ranges for the low- $E_{T}^{\text {jet }}$ cuts are as in the previous $H 1$ analysis [5] and for the high- $E_{T}^{j e t}$ cuts are chosen as in the ZEUS analysis with two exceptions. In the ZEUS analysis the maximal cut on $Q^{2}$ is larger and the data are taken in an extended $y$-range. The definition of the various variables can be found in the $\mathrm{H} 1$ and ZEUS publications $[5,6]$. Very important is the cut on $x_{\mathbb{P}}$. It is kept small in both analyses in order for the pomeron exchange to be dominant. In the experimental analysis as well as in the NLO calculations, jets are defined with the inclusive $k_{T^{-}}$ cluster algorithm $[19,20]$ in the laboratory frame. At least two jets are required with the respective cuts on $E_{T}^{j e t 1}$ and $E_{T}^{j e t 2}$, where $E_{T}^{j e t 1(2)}$ refers to the jet with the largest (second largest) $E_{T}^{j e t}$.

Before we confront the calculated cross sections with the experimental data, we correct them for hadronization effects. The hadronization corrections are calculated by means of the LO RAPGAP Monte Carlo generator. The factors for the transformation of jets made up of stable hadrons to parton jets were supplied by the H1 collaboration [17]. Our calculations are done with the 'H1 2006 fit B' [4] DPDFs, since they give smaller diffractive dijet cross sections than with the 'H1 2006 fit A'. We then take $n_{f}=4$ with
$\Lambda_{\overline{\mathrm{MS}}}^{(4)}=0.347 \mathrm{GeV}$, which corresponds to the value used in the DPDFs 'H1 2006 fit A, B' [4]. For the photon PDFs we have chosen the NLO GRV parameterization transformed to the $\overline{\mathrm{MS}}$ scheme [21].

As it is clear from the discussion of the various preliminary analyses of the $\mathrm{H} 1$ and ZEUS collaborations, there are two questions which we would like to answer from the comparison with the recent $\mathrm{H} 1$ and the ZEUS data. The first question is whether a suppression factor, which differs substantially from one, is needed to describe the data. The second question is whether the data are also consistent with a suppression factor applied to the resolved cross section only. For both suppression models it is also of interest, whether the resulting suppression factors are universal, i.e. whether they are independent of the kinematic variables of the process. To give an answer to these two questions we calculated first the cross sections with no suppression factor ( $R=1$ in the following figures) with a theoretical error obtained from varying the common scale of renormalization and factorization by factors of 0.5 and 2 about the default value (highest $E_{T}^{j e t}$ ). In a second step we show the results for the same differential cross sections with a global suppression factor, adjusted to $d \sigma / d E_{T}^{j e t 1}$ at the smallest 

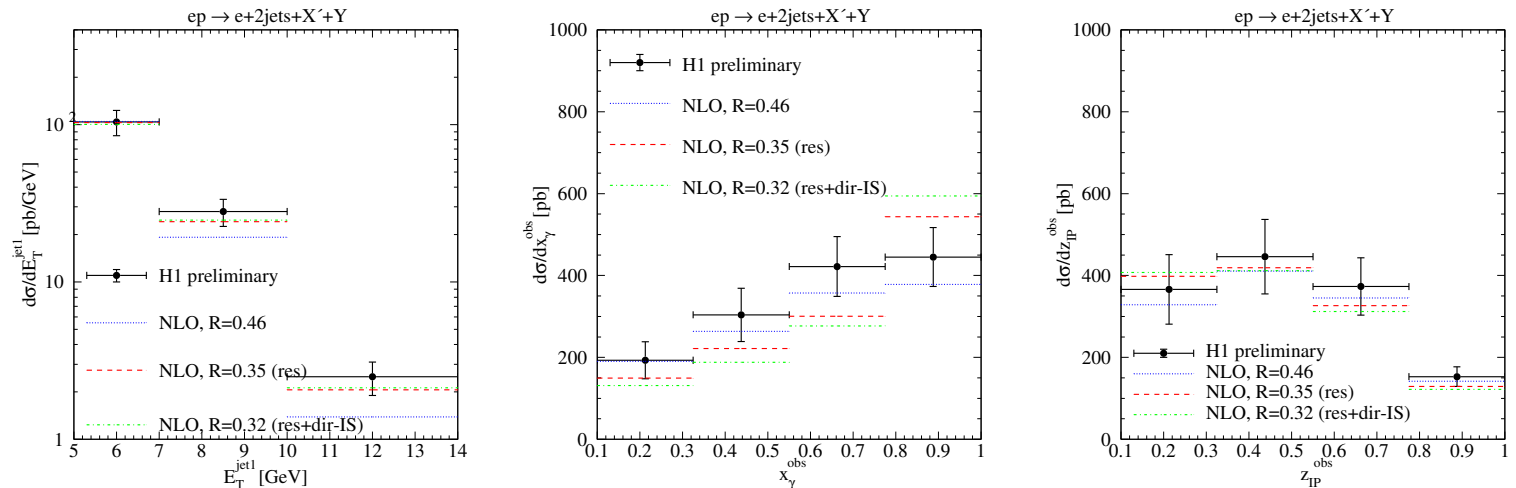

Figure 2. Differential cross sections for diffractive dijet photoproduction as measured by H1 with low- $E_{T}^{j e t}$ cuts and compared to NLO QCD with global, resolved, and resolved/direct-IS suppression. (a), (b), and (c) as in Fig. 1 (color online).

$E_{T}^{j e t 1}$-bin. As in the experimental analysis [17], we have considered the differential cross sections in the variables $x_{\gamma}^{o b s}, z_{\mathbb{P}}^{o b s}, \log _{10}\left(x_{\mathbb{P}}\right), E_{T}^{j e t 1}, M_{X}$, $M_{12}, \bar{\eta}^{j e t s},\left|\Delta \eta^{j e t s}\right|$ and $W[18]$. Here we show only a selection, i.e. the cross sections as a function of $E_{T}^{j e t 1}, x_{\gamma}^{o b s}$ and $z_{\mathbb{P}}^{o b s}$. For the low- $E_{T}^{j e t}$ cuts, the resulting suppression factor is $R=0.46 \pm 0.08$, which gives in the lowest $E_{T}^{\text {jet1 }}$-bin a cross section equal to the experimental data point. The error comes from the combined experimental statistical and systematic error. The theoretical error due to the scale variation is taken into account when comparing to the three distributions. The results of this comparison are shown in Figs. 1a-c. With the exception of Fig. 1a, where the comparison of $d \sigma / d E_{T}^{j e t 1}$ is shown, the other two plots are such that the data points lie outside the error band based on the scale variation for the unsuppressed case. However, the predictions with suppression $R=0.46$ agree nicely with the data inside the error bands from the scale variation. Most of the data points even agree with the $R=0.46$ predictions inside the much smaller experimental errors. In $d \sigma / d E_{T}^{j e t 1}$ (see Fig. 1a) the predictions for the second and third bins lie outside the data points with their errors. For $R=1$ and $R=0.46$ this cross section falls off more strongly with increasing $E_{T}^{j e t 1}$ than the data, the normalization being of course about two times larger for $R=1$. In particular, the third data point agrees with the $R=1$ prediction. This means that the suppression decreases with increasing $E_{T}^{\text {jet } 1}$ (see also Fig. 5 below). This behavior was already apparent when we analyzed the first preliminary H1 data $[1,2]$. Such a behavior points in the direction that a suppression of the resolved cross section only would give better agreement with the data, as we shall see below, since the relative contribution of the resolved part diminishes at large $E_{T}^{j e t}$ as compared to the direct part and therefore the combined cross section is automatically suppressed less at large $E_{T}^{j e t}$. The survival probability $R=0.46 \pm 0.08$ agrees with the result in [17], which quotes $R=0.51 \pm 0.01$ (stat.) \pm 0.10 (syst.), determined by fitting the integrated cross section. From our comparison we conclude that the low- $E_{T}^{\text {jet }}$ data show a global suppression of the order of two in complete agreement with the results $[1,2,5]$ based on earlier preliminary and final $\mathrm{H} 1$ data [5].

Next we want to answer the second question, whether the data could be consistent with a suppression of the resolved component only. For this purpose we have calculated the cross sections in two additional versions: (i) suppression of the resolved cross section and (ii) suppres- 

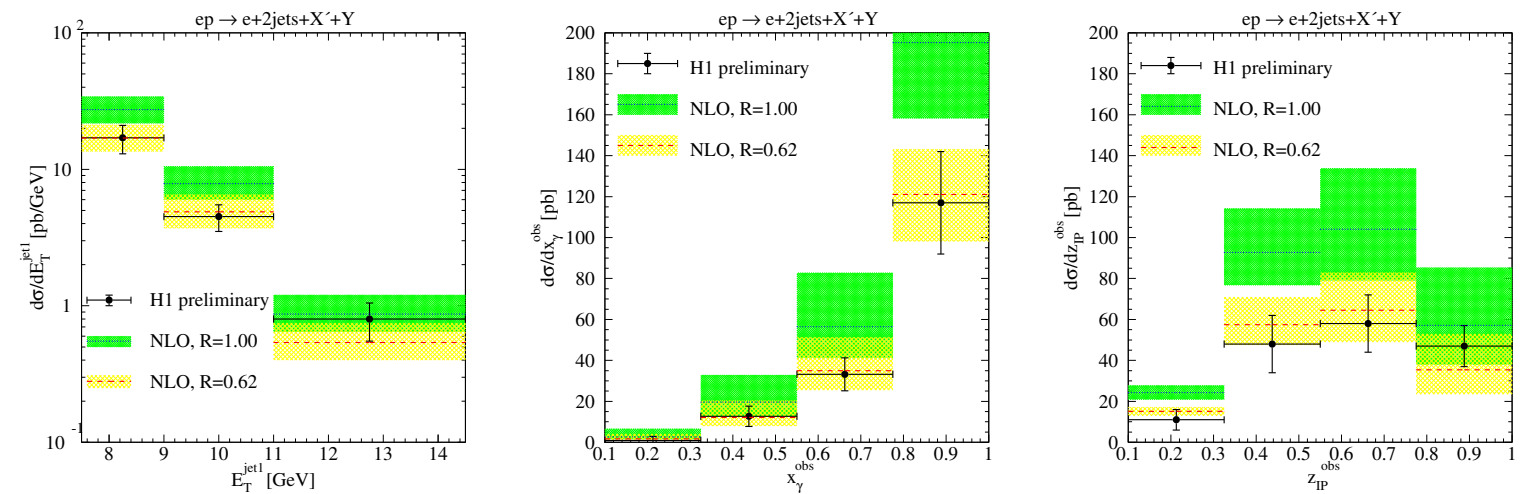

Figure 3. Differential cross sections for diffractive dijet photoproduction as measured by H1 with high$E_{T}^{\text {jet }}$ cuts and compared to NLO QCD without $(R=1)$ and with $(R=0.62)$ global suppression. (a), (b) and (c) as in Fig. 1 (color online).
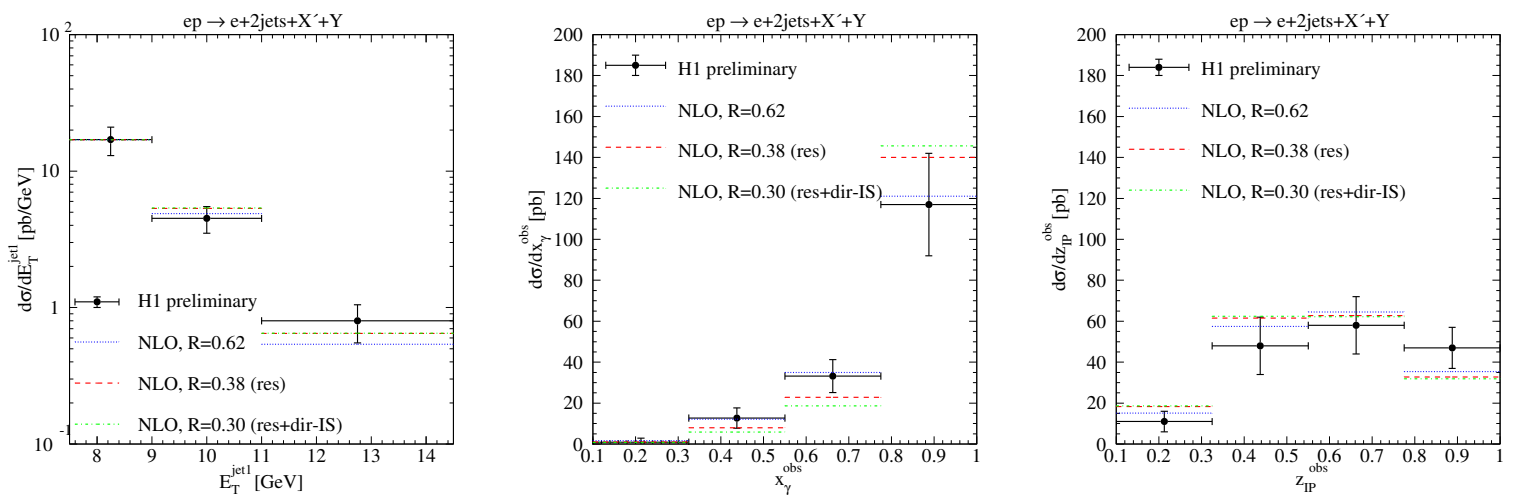

Figure 4. Differential cross sections for diffractive dijet photoproduction as measured by H1 with high$E_{T}^{\text {jet }}$ cuts and compared to NLO QCD with global, resolved, and resolved/direct-IS suppression. (a), (b) and (c) as in Fig. 1 (color online).

sion of the resolved cross section plus the NLO direct part which depends on the factorization scale at the photon vertex [10]. The suppression factors needed for the two versions will, of course, be different. We determine them again by fitting the measured $d \sigma / d E_{T}^{j e t 1}$ for the lowest $E_{T}^{\text {jet } 1}$-bin (see Fig. 2a). Then, the suppression factor for version (i) is $R=0.35$ (denoted res in the figures), and for version (ii) it is $R=0.32$ (denoted res+dir-IS). The results for $d \sigma / d E_{T}^{j e t 1}$, $d \sigma / d x_{\gamma}^{o b s}$ and $d \sigma / d z_{\mathbb{P}}^{o b s}$ are shown in Figs. 2a-c, while the six other distributions can be found in [18]. We also show the global (direct and resolved) suppression prediction with $R=0.46$ already shown in Figs. 1a-c. For the cross section as a function of $z_{\mathbb{P}}^{o b s}$, the agreement with the global suppression $(R=0.46)$ and the resolved suppression $(R=0.35$ or $R=0.32)$ is comparable. For $d \sigma / d E_{T}^{j e t 1}$, the agreement improves considerably for the resolved suppression 

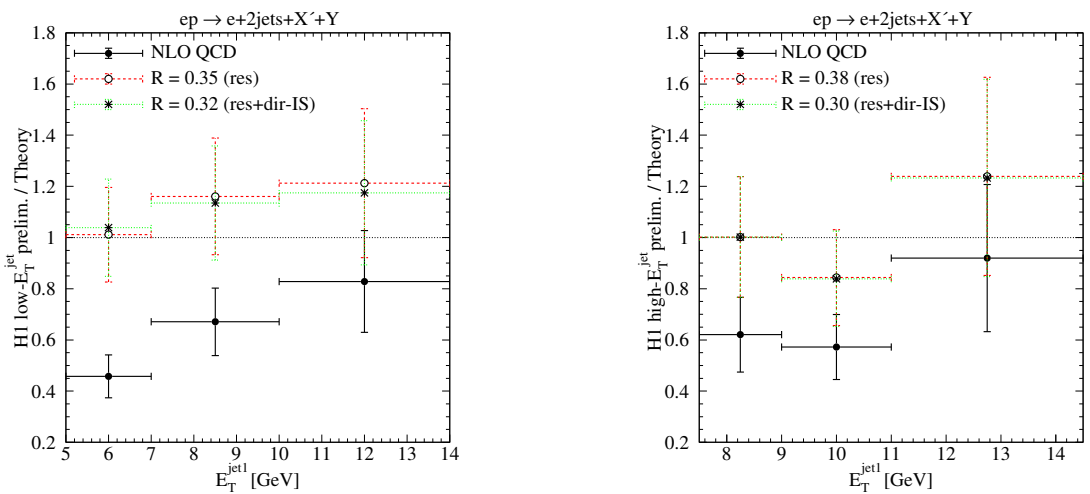

Figure 5. Ratio of the $E_{T}^{\text {jet1 }}$-distribution as measured by $\mathrm{H} 1$ with low- $E_{T}^{\text {jet }}$ (left) and high- $E_{T}^{\text {jet }}$ cuts (right) to the NLO QCD prediction without (full), with resolved-only (dashed), and with additional direct IS suppression (dotted) (color online).

only (note the logarithmic scale in Fig. 2a). The global suppression factor could, of course, be $E_{T^{-}}$ dependent, although we see no theoretical reason for such a dependence. For $d \sigma / d x_{\gamma}^{o b s}$, which is usually considered as the characteristic distribution for distinguishing global versus resolved suppression, the agreement with resolved suppression does not improve. Unfortunately, this cross section has the largest hadronic corrections of the order of $(25-30) \%$ [17]. Second, also for the usual photoproduction of dijets the comparison between data and theoretical results has similar problems in the large $x_{\gamma}^{o b s}$-bin [22], although the $E_{T}^{j e t}$-cut is much larger there. In total, we are tempted to conclude from the comparisons in Figs. 2a-c that the predictions with a resolvedonly (or resolved+direct-IS) suppression are consistent with the new low- $E_{T}^{\text {jet }} \mathrm{H} 1$ data [17] and the survival probability is $R=0.35$ (only resolved suppression) and $R=0.32$ (resolved plus directIS suppression), respectively.

The same comparison of the high- $E_{T}^{j e t}$ data of $\mathrm{H} 1$ [17] with the various theoretical predictions is shown in the following figures. The global suppression factor is obtained again from a fit to the cross sections for the smallest $E_{T}^{\text {jet } 1}$-bin. It is equal to $R=0.62 \pm 0.16$, again in agreement with the $\mathrm{H} 1$ result $R=0.62 \pm 0.03$ (stat.) \pm 0.14 (syst.)
[17]. The same cross sections as for the low- $E_{T}^{j e t}$ comparison are shown in Figs. 3a-c for the two cases $R=1$ (no suppression) and $R=0.62$ (global suppression), while the six others can again be found in [18]. As before with the exception of $d \sigma / d E_{T}^{j e t 1}$ and $d \sigma / d M_{12}$ (not shown), most of the data points lie outside the $R=1$ results with their error bands and agree with the suppressed prediction with $R=0.62$ inside the respective errors. However, compared to the results in Figs. 1a-c the distinction between the $R=1$ band and the $R=0.62$ band and the data is somewhat less pronounced, since the larger suppression factor is larger now. We also tested the prediction for the resolved (resolved+direct-IS) suppression, which is shown in Figs. 4a-c. The suppression factor fitted to the smallest bin came out as $R=0.38$ (res) and $R=0.30$ (res+dir-IS), which are almost equal to the corresponding suppression factors derived from the low- $E_{T}^{j e t}$ data. In most of the comparisons it is hard to observe any preference for the global against the pure resolved (resolved+direct-IS) suppression. We remark that the suppression factor for the global suppression is increased by $35 \%$, if we go from the low- $E_{T}^{j e t}$ to the high- $E_{T}^{j e t}$ data, whereas for the resolved suppression this increase is only $9 \%$. Under the assumption that the suppression fac- 

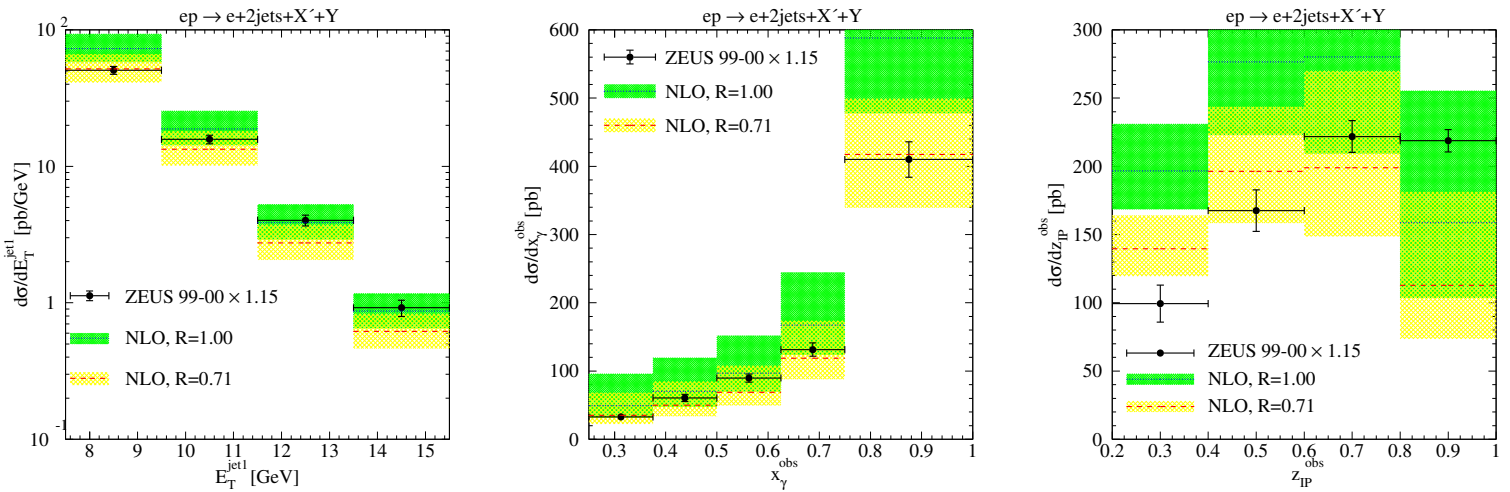

Figure 6. Differential cross sections for diffractive dijet photoproduction as measured by ZEUS and compared to NLO QCD without $(R=1)$ and with $(R=0.71)$ global suppression. (a), (b) and (c) as in Fig. 1 (color online).

tor should not depend on $E_{T}^{j e t 1}$, i.e. being universal and only dependent on the HERA c.m. energy, we would conclude that the resolved suppression would be preferred, as can also be seen from Fig. 5. A global suppression is definitely observed also in the high- $E_{T}^{\text {jet }}$ data and the version with resolved suppression explains the data almost as well as with the global suppression.

In Fig. 5 we show the ratio of the $E_{T}^{\text {jet1 }}$ distribution as measured by $\mathrm{H} 1$ to the NLO QCD prediction without (full), with resolved-only (dashed), and with additional direct IS suppression (dotted) as a function of $E_{T}^{j e t 1}$. Within the experimental errors, obviously only the former, but not the latter are $E_{T}^{j e t}$-dependent. In the case of resolved-only and the case with additional direct IS suppression these ratios lie inside the experimental errors near one over the whole $E_{T}^{j e t 1}$ range.

\section{Comparison with ZEUS data}

In this section I shall compare the predictions with the final analysis of the ZEUS data, which was published this year [6], in order to see whether they are consistent with the large$E_{T}^{j e t}$ data of H1. The kinematic cuts [6] are almost the same as in the high- $E_{T}^{j e t} \mathrm{H} 1$ measure- ments. The only major difference to the $\mathrm{H} 1$ cuts is the larger range in the variable $y$. Therefore the ZEUS cross sections will be larger than the corresponding $\mathrm{H} 1$ cross sections. The constraint on $M_{Y}$ is not explicitly given in the ZEUS publication [6]. They give the cross section for the case that the diffractive final state consists only of the proton. For this they correct their measured cross section by subtracting in all bins the estimated contribution of a proton-dissociative background of $16 \%$. When comparing to the theoretical predictions they multiply the cross section with the factor 0.87 in order to correct for the protondissociative contributions, which are contained in the DPDFs 'H1 2006 fit A' and 'H1 2006 fit B' by requiring $M_{Y}<1.6 \mathrm{GeV}$. We do not follow this procedure. Instead we leave the theoretical cross sections unchanged, i.e. they contain a protondissociative contribution with $M_{Y}<1.6 \mathrm{GeV}$ and multiply the ZEUS cross sections by 1.15 to include the proton-dissociative contribution. This means that the so multiplied ZEUS cross sections should have the same proton dissociative contribution as is in the DPDF fits of H1 [4]. Since the ZEUS collaboration did measurements only for the high- $E_{T}^{j e t}$ cuts, $E_{T}^{j e t 1(2)}>7.5(6.5) \mathrm{GeV}$, we can only compare to those. In this comparison we shall follow the same strategy as before. 

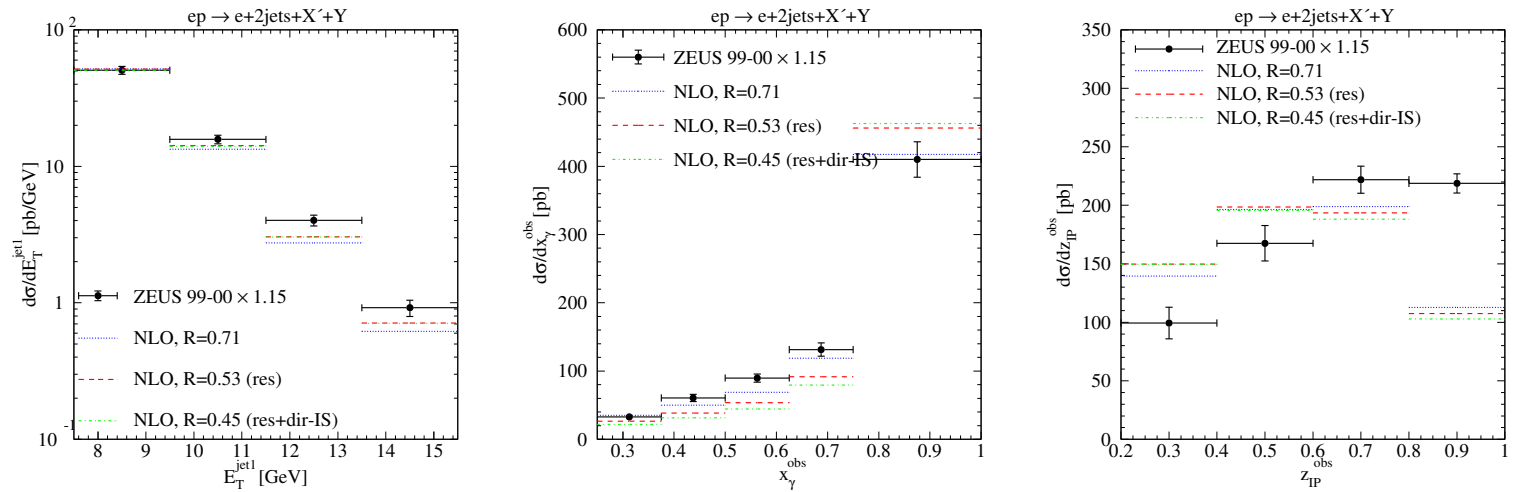

Figure 7. Differential cross sections for diffractive dijet photoproduction as measured by ZEUS and compared to NLO QCD with global, resolved, and resolved/direct-IS suppression. (a), (b) and (c) as in Fig. 1 (color online).

We first compared to the predictions with no suppression $(R=1)$ and then determine a suppression factor by fitting $d \sigma / d E_{T}^{j e t 1}$ at the smallest $E_{T}^{j e t 1}$-bin. Then we compared to the cross sections as a function of the seven observables $x_{\gamma}^{o b s}$, $z_{\mathbb{P}}^{o b s}, x_{\mathbb{P}}, E_{T}^{j e t 1}, y, M_{X}$ and $\eta^{j e t 1}$ instead of the nine variables in the $\mathrm{H} 1$ analysis. The distribution in $y$ is equivalent to the $W$-distribution in [17]. The theoretical predictions for these differential cross sections with no suppression factor $(R=1)$ are shown in Figs. 6a-g of [15], together with their scale errors and compared to the ZEUS data points. A selection is shown in Fig. 6. Except for the $x_{\gamma}^{\text {obs }}$ - and $E_{T}^{j e t 1}$-distributions, most of the data points lie outside the theoretical error bands for $R=1$. In particular, in Figs. 6b, c, e, $\mathrm{f}$ and $\mathrm{g}$, of Ref. [15] most of the points lie outside. This means that most of the data points disagree with the unsuppressed prediction. Next, we determine the suppression factor from the measured $d \sigma / d E_{T}^{j e t 1}$ at the lowest $E_{T}^{j e t 1}$-bin, $7.5 \mathrm{GeV}$ $<E_{T}^{j e t 1}<9.5 \mathrm{GeV}$, and obtain $R=0.71$. This factor is larger by a factor of 1.15 than the suppression factor from the analysis of the high- $E_{T}^{\text {jet }}$ data from H1. Taking the total experimental error of $\pm 7 \%$ from the experimental cross section $d \sigma / d E_{T}^{j e t 1}$ in the first bin into account, the ZEUS suppression factor is $0.71 \pm 0.05$ to be compared to $0.62 \pm 0.14$ in the $\mathrm{H} 1$ analysis [17], so that both suppression factors agree inside the experimental errors. Actually the ZEUS cross sections should be reduced by $13 \%$. This follows from a comparison of the diffractive inclusive data obtained by the ZEUS collaboration with the large rapidity gap method with the corresponding inclusive diffractive data of the $\mathrm{H} 1$ collaboration [4], where the latter data include a dissociative proton contribution with the cut $M_{Y}<1.6 \mathrm{GeV}$ [23].

If we now check how the predictions for $R=$ 0.71 compare to the data points inside the theoretical errors, we observe from Figs. 6a-g of Ref. [15] that, with the exception of $d \sigma / d z_{\mathbb{P}}^{o b s}$ and $d \sigma / d E_{T}^{j e t} 1$, most of the data points agree with the predictions. This is quite consistent with the H1 analysis (see above) and leads to the conclusion that also the ZEUS data agree much better with the suppressed predictions than with the unsuppressed prediction. In particular, the global suppression factor agrees perfectly with the global suppression factor obtained from the analysis of the $\mathrm{H} 1$ data, if we take into account the $13 \%$ reduction of the cross section mentioned above.

Similarly as in the previous section we compared the ZEUS data also with the assumption that the suppression results only from the resolved cross section. Here, we consider again (i) 

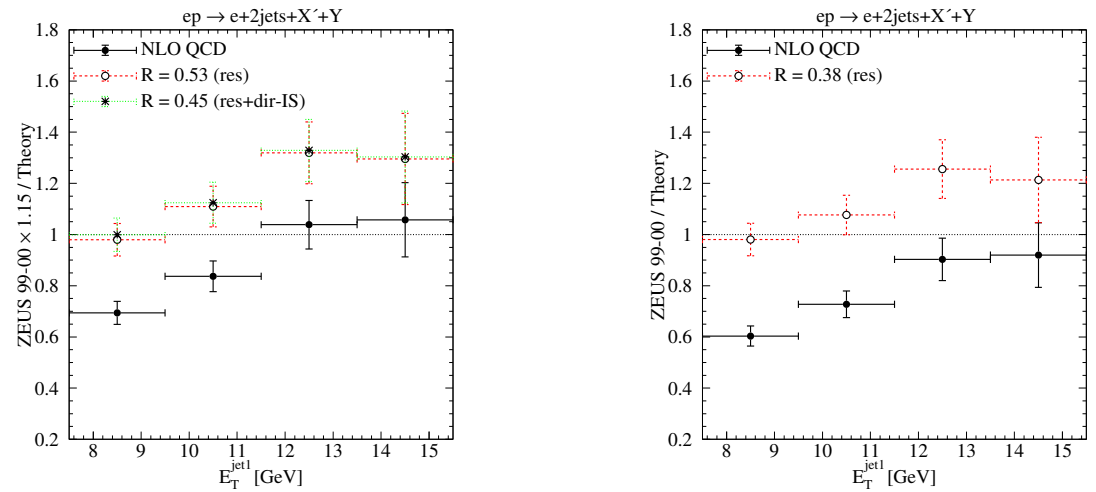

Figure 8. Ratio of the $E_{T}^{\text {jet1 }}$-distribution as measured by ZEUS with original normalization (left) and with reduced normalization by the factor 1.15 (right) to the NLO QCD prediction without (full), with resolved-only (dashed) and with additional IS direct suppression (dotted) (color online).

only resolved suppression (res) and (ii) resolved plus direct suppression of the initial-state singular part (res+dir-IS). For these two models we obtain the suppression factors $R=0.53$ and $R=0.45$, respectively, where these suppression factors are again obtained by fitting the data point at the first bin of $d \sigma / d E_{T}^{j e t 1}$. The comparison to the global suppression with $R=0.71$ and to the data is shown in Figs. 7a-g of [15] and a selection in Fig. 7. In general, we observe that the difference between global suppression and resolved suppression is small, i.e. the data points agree with the resolved suppression as well as with the global suppression. For the ZEUS data we have also plotted the ratio of the measured cross section $d \sigma / d E_{T}^{j e t 1}$ to the NLO prediction without (full), with resolved-only (dashed), and with additional direct-IS suppression (dotted) in Fig. 8 (left side). The full points show the dependence on $E_{T}^{j e t 1}$ as for the H1 data. Unfortunately for the dashed and dotted points this dependence is not very much reduced, but all the ratios now lie somewhat nearer to one. This looks even better, when we reduce the normalization of the measured ZEUS cross section by $15 \%$, where the suppression factor is $R=0.38$ (for resolved suppression only) which agrees perfectly with the suppresion factor obtained from the H1 high $E_{T}^{j e t}$ data above.
The corresponding ratios are also shown in Fig. 8 (right side).

In all the suppression factors reported in this paper I have included only the experimental error, which comes from the error of $d \sigma / d E_{T}^{j e t 1}$ at the smallest $E_{T}^{j e t 1}$-bin. In addition, there exists also the theoretical error, which usually is taken from the scale variation of the respective cross sections. These errors can be read off from the plots for $d \sigma / d E_{T}^{j e t 1}$ shown above and would be relevant for the question, whether the suppression factors would be below one when all the errors are taken into account. For the low- $E_{T}^{j e t 1}$ data from $\mathrm{H} 1$ we get the supression factor $\mathrm{R}=0.46 \pm 0.14$, and for the high- $E_{T}^{j e t 1}$ data the suppression factor is $\mathrm{R}=0.62 \pm 0.20$ when both errors, the experimental one and the theoretical one are included. For the ZEUS data the suppression factor is the same with a somewhat smaller total error, since their experimental error is smaller, the theoretical error being the same as for H1. This means that all the global suppression factors are smaller than one inside their errors.

\section{Conclusion}

In conclusion, we found that most of the data points of diffractive dijet photoproduction in the 
latest $\mathrm{H} 1$ analyses with low- and high- $E_{T}^{\text {jet }}$ cuts and in the final ZEUS analysis with the same high- $E_{T}^{j e t}$ cuts disagree with NLO QCD predictions within experimental and theoretical errors. When global factorization breaking is assumed in both the direct and resolved contributions, the resulting suppression factor would have to be $E_{T}^{j e t}$-dependent, i.e. would not be universal for all diffractive dijet photoproduction data. Suppressing only the resolved or in addition the direct initial-state singular contribution by about a factor of three, as motivated by the proof of factorization in point-like photon-hadron scattering and predicted by absorptive models [14], the agreement between theory and data is at least as good as for global suppression, and no $E_{T}^{j e t}$ dependence of the survival probability is seen.

\section{Acknowlegdements}

It is a pleasure to thank Michael Klasen for his continued collaboration and Karel Cerny for information about the preliminary data.

\section{REFERENCES}

1. M. Klasen and G. Kramer, Proc. of the 12th Int. Workshop on Deep Inelastic Scattering, Strbske Pleso, Slovakia, 14-18 April (2004) 492.

2. M. Klasen and G. Kramer, Eur. Phys. J. C 38 (2004) 93.

3. H1 Collaboration, paper 987, contributed to the 31st Int. Conf. on High Energy Physics (ICHEP 02), Amsterdam (2002); paper 087, contributed to the Int. Europhysics Conf. on High Energy Physics (EPS 03), Aachen (2003) and F.-P. Schilling, Eur. Phys. J. C 33 (2004) s530.

4. A. Aktas et al. [H1 Collaboration], Eur. Phys. J. C 48 (2006) 715.

5. A. Aktas et al. [H1 Collaboration], Eur. Phys. J. C 51 (2007) 549.

6. S. Chekanov et al. [ZEUS Collaboration], Eur. Phys. J. C 55 (2008) 177.

7. J.C. Collins, Phys. Rev. D 57 (1998) 3051; J. Phys. G 28, 1069 (2002).
8. A.A. Affolder et al. [CDF Collaboration], Phys. Rev. Lett. 84 (2000) 5043.

9. M. Klasen, Rev. Mod. Phys. 74 (2002) 1221.

10. M. Klasen and G. Kramer, J. Phys. G 31 (2005) 1391.

11. M. Klasen, Proc. of the Ringberg Workshop "NEW TRENDS IN HERA PHYSICS 2005", World Scientific (2006) 293.

12. A. Bruni, M. Klasen, G.Kramer and S. Schaetzel, Contribution to the Workshop on the Implications of HERA for LHC Physics, CERN, Geneva, Switzerland, 26-27 March (2004), arXiv:hep-ph/0509202.

13. M. Klasen and G. Kramer, AIP Conf. Proc. 792 (2005) 444.

14. A.B. Kaidalov V.A. Khoze, A.D. Martin and M.G. Ryskin, Phys. Lett. B 567 (2003) 61.

15. M. Klasen and G. Kramer, Mod. Phys. Lett. A 23 (2008) 1885.

16. L. Schoeffel, R. Sacchi and A. Bunyatian, Contributions in these proceedings.

17. K. Cerny [H1 Collaboration], Proc. of 16th Int. Workshop on Deep Inelastic Scattering, London, England, 7-11 April (2008), http://dx.doi.org/10.3360/dis.2008.69.

18. M. Klasen and G. Kramer, DESY 08-109, LPSC 08-113, to be published.

19. S.D. Ellis and D.E. Soper, Phys. Rev. D 48 (1993) 3160.

20. S. Catani, Y.L. Dokshitzer, M.H. Seymour and B.R. Webber, Nucl. Phys. B 406 (1993) 187.

21. M. Glück, E. Reya and A. Vogt, Phys. Rev. D 46 (1992) 1973.

22. A. Aktas et al. [H1 Collaboration], Phys. Lett. B 639 (2006) 21.

23. L. Schoeffel, Contribution in these proceedings. 